\title{
Chefarzt unter Druck
}

\author{
Jürg Bänninger \\ Dr. med., ehem. Leitungsausschuss ReMed
}

\section{Chefarzt Orthopädie}

Der Kollege eröffnete das erste Telefongespräch mit ReMed mit dem bescheidenen Anliegen, «einfach etwas deponieren zu dürfen». Nach sieben Jahren als Chefarzt eines Regionalspitals ist er mit allen Wassern gewaschen und verfügt über eine robuste Resilienz. Die jüngsten bekanntgegebenen «Umstrukturierungen» am Spital und auf seiner Abteilung lassen ihm jedoch jetzt keine Ruhe - diese betreffen nicht mehr nur ihn selbst, sondern letztlich und am meisten das vulnerabelste Glied - die Patientinnen und Patienten. Ehrlich empört meint der Kollege: «Ich bin jetzt 59 Jahre alt, und ich glaube, ich muss da weg - muss diese Stelle künden. Ich habe unzählige Male als Chefarzt meine eigenen Vorstellungen, Anliegen und Werte beiseitegeschoben und mich damit arrangiert, dass meine Argumente einfach in den Wind geschlagen werden. Diese jüngsten Entwicklungen jetzt kann ich so allerdings nicht mehr akzeptieren: Sie betreffen direkt die Qualität der Behandlung unserer Patienten.»

\section{Nachfolgeregelung gut geplant}

Ein langjähriger leitender Arzt auf der Orthopädie an diesem mittelgrossen Spital wurde als Chefarzt an ein anderes Spital gewählt. Der Chef lässt ihn ungern ziehen, aber es freut ihn auch. Vorsorglich sorgte er rechtzeitig dafür, dass die Nachfolge für den leitenden Arzt geregelt werden kann. Er tat das Naheliegendste und zog dafür die motivierte, engagierte und ideal geeignete Oberärztin nach.

\section{Die Spitalleitung hat aber ganz andere Pläne}

"Alles schien auf guten Wegen, als wie ein Blitz aus heiterem Himmel der einzige Arzt in der Spitalleitung (alle anderen Mitglieder kommen aus der Wirtschaft) mich an einem späten Abend aufsuchte und mir mitteilte, es sei beschlossen worden, im Hinblick auf diese Nachfolge die Verträge mit den leitenden Ärzten grundsätzlich neu zu regeln. In Zukunft hätten die Leitenden ein deutlich kleineres Fixum und ein variables, umsatzabhängiges Salär. Dies ist eine für die begabte
Orthopädin absolut inakzeptable Option. Zudem verlange die Spitalleitung, dass auf allen Abteilungen ein Belegarztsystem eingerichtet und der Dienst auf diese auswärtigen Kollegen ausgedehnt werde. Der Kollege hätte alles versucht, um sich für mich einzusetzen, aber es sei aus finanziellen Überlegungen nicht mehr daran zu rütteln. Vielleicht könne ich ja auch Vorteile daraus ziehen, denn immerhin entfalle mir durch dieses Belegarztdienstsystem ein grosser Brocken an Verantwortung.

Ökonomen ohne medizinisches Wissen und ohne jegliche Erfahrung in der Führung einer medizinischen Abteilung diktieren Chefärzten zunehmend die Rahmenbedingungen ihrer Arbeit.

Ich war völlig konsterniert, denn Verantwortung zu tragen und mich zu engagieren ist für mich keine Last, sondern dazu fühle ich mich als Chef berufen. Aber hier wurde mir Verantwortung und Einfluss auf die Führung meiner Abteilung von Spitalmanagern schlicht aus den Händen gerissen. Dies von Leuten, die keine Ahnung von Medizin und nichts als Zahlen im Kopf haben. Dies alles ohne Rückfrage, ohne ein vorangehendes Gespräch. Die Spitalleitung reichte mir noch nach, wie viel Geld das Spital durch diese Umstrukturierung einzusparen plant. Offensichtlich sind sich diese Führungsleute nicht im Klaren, was bei einer solchen Hauruck-Übung alles zerstört wird.»

\section{Der Einfluss auf die Führungsmöglich- keiten und das Arbeitsklima ist enorm}

«Das hat weitreichende Konsequenzen: Wir sind ein gut eingespieltes, hochengagiertes Team, und es herrschte

\footnotetext{
ReMed ist für Sie da

Brauchen Sie oder jemand aus Ihrem Umfeld professionelle Hilfe? Wenden Sie sich an ReMed: Das Unterstützungsnetzwerk für Ärztinnen und Ärzte respektiert das Arztgeheimnis und berät Sie kompetent. Auch bei anderen beruflichen und persönlichen Krisen kann Ihnen ReMed Lösungswege aufzeigen. Dieses Angebot gilt auch für Personen aus dem Umfeld von Ärztinnen und Ärzten. 24 Stunden am Tag. Die ärztlichen Beratenden melden sich innerhalb von 72 Stunden: www.swiss-remed.ch, help[at] swissremed.ch, Tel. 0800073633.
} 


\section{Coaching-Gruppen}

Die Coaching-Gruppen bieten den idealen Rahmen für einen vertrauensvollen Austausch über sämtliche schwierigen beruflichen Themen, z.B. den Umgang mit Kolleginnen und Kollegen sowie Vorgesetzten, Ängste oder Belastungsgrenzen. Das Ziel der Coaching-Gruppen ist es, unter fachlich kompetenter Anleitung in ein kollegiales Gespräch zu kommen und sich gegenseitig zu bestärken. Das Angebot richtet sich an Assistenz- und Oberärztinnen und -ärzte am Spital und in Praktika sowie an niedergelassene Medizinerinnen und Mediziner. Es schliesst Teilnehmende sämtlicher Fachrichtungen ein. Zurzeit werden Coaching-Gruppen in Bern, Zürich und St. Gallen durchgeführt. Genauere Informationen finden Sie unter www.swiss-remed.ch $\rightarrow$ Weitere Angebote $\rightarrow$ Coaching-Gruppen.

auf der Abteilung hohe Zufriedenheit bei allen. Das schätzen auch die Patienten, die Umfragen zeigen immer eine grosse Patientenzufriedenheit. Betont wird besonders die persönliche Betreuung durch den zuständigen Arzt.

Mit dem Pflegeteam hat sich über die Jahre eine fruchtbare und qualitativ hervorragende Zusammenarbeit entwickelt. Durch die Einführung eines belegärztlich organisierten Dienstes geht diese persönliche und verbindliche Zusammenarbeit im Team verloren. Ich suchte etliche Male das Gespräch mit der Spitalleitung und wehrte mich erfolglos. Schliesslich hat mir der CEO gedroht, man könne auch die gesamte Orthopädie am Spital mit Belegärzten organisieren!»

\section{Korrespondenz:}

ReMed

Sekretariat

Postfach 55

CH-3000 Bern 15

www.swiss-remed.ch

info[at]swiss-remed.ch

\section{Es ist wichtig, zu merken, dass man nicht alleine ist}

ReMed als Unterstützungsnetzwerk bot diesem Chefarzt zunächst die Möglichkeit einer vertraulichen Aussprache. Erst im Verlaufe des Gesprächs merkte der
Kollege, wie sehr ihn die Situation am Spital schon lange belastete. Er wurde von ReMed ernst genommen, und sein Gegenüber wusste genau, wovon er sprach. Er konnte erfahren, dass er nicht alleine war mit seinen Sorgen. Lange versuchte er, sein Umfeld zu schonen, und schluckte alles hinunter. Nun merkte er, dass allein schon das darüber Sprechen guttat. ReMed kann in diesem Fall nicht direkt Einfluss nehmen. Aber für das weitere Vorgehen gegenüber der Spitalleitung und die Planung seiner eigenen beruflichen Zukunft taten sich neue Wege auf, z.B. sich ab und zu bei einer erfahrenen und vertrauensvollen Person Unterstützung zu holen. ReMed vermittelte dem Chefarzt ein geeignetes Coaching, das er nach Gutdünken beanspruchen kann. Im Bewusstsein, dass man als Chef per se in vielen Belangen auf sich alleine gestellt ist, stand ihm jetzt mit dem von uns vorgeschlagenen Coaching eine Möglichkeit zur Verfügung, die er ausprobieren konnte.

\section{ReMed-Intervisionen für Erstberatende und Netzwerkmitglieder}

Neben den Unterstützungsangeboten für ratsuchende Ärztinnen und Ärzte führt ReMed seit 2009 auch regionale Intervisionen zum Erfahrungsaustausch für Kolleginnen und Kollegen durch, die Ärztinnen und Ärzte als Patienten betreuen. Diese ermöglichen Vernetzung und Bildung von Peer-Groups (jeweils 6-8 Teilnehmer, 2-3 Treffen pro Jahr), welche gemeinsam Fallfragen zu Mentoring, Coaching, Beratung, Therapie oder anderen Aspekten (juristisch, versicherungsrechtlich etc.) erarbeiten. Setzen Sie sich mit uns in Verbindung, nehmen Sie an einer Sitzung teil und lernen Sie unsere Arbeit kennen. Kontakt und Anmeldung: Dr. med. Sabine Werner, Mitglied Leitungsausschuss ReMed, dr.s.werner[at]hin.ch

Mögliche nächste Daten 2019: 28. Februar, 7. März, 4. April, 16. Mai, 13. Juni, 26. September, 14. November und 21. November, jeweils 14-18 Uhr, in Zürich. 